\title{
Effects of residual and tunable strain in thin films of $\mathrm{La}_{0.7} \mathrm{Ba}_{0.3} \mathrm{MnO}_{3}$
}

\author{
J. F. Wang, Y. C. Jiang, Z. P. Wu, and J. Gao ${ }^{\text {a) }}$ \\ Department of Physics, The University of Hong Kong, Pokfulam Road, Hong Kong
}

(Presented 17 January 2013; received 4 November 2012; accepted 19 December 2012; published online 22 March 2013)

\begin{abstract}
The effects of residual and tunable strain in thin films of $\mathrm{La}_{0.7} \mathrm{Ba}_{0.3} \mathrm{MnO}_{3}$ (LBMO) were investigated. Different residual strains were achieved by depositing LBMO films with various thicknesses on $0.7 \mathrm{PbMg}_{1 / 3} \mathrm{Nb}_{2 / 3} \mathrm{O}_{3}-0.3 \mathrm{PbTiO}_{3}\left(\mathrm{PMN}-\mathrm{PT}\right.$ ) and $\mathrm{SrTiO}_{3}$ (STO). For LBMO on PMNPT, when the thickness changes the resistance evolves dramatically. All samples on STO have a clear metal-to-insulator transition near room temperature. The tunable strain was modulated through the converse piezoelectric effects of PMN-PT. Clear voltage-controlled resistance modulations $\left(|\Delta \mathrm{R}| / \mathrm{R}_{\max } \sim 90 \%\right)$ were observed in LBMO/PMN-PT. The magnitude and nonlinearity of elastoresistance are the largest in the thinnest sample. $|\Delta \mathrm{R}| / \mathrm{R}$ decreases as the thickness increases and is still substantial even the film is as thick as $150 \mathrm{~nm}$. For thicker samples, the nonlinearity becomes weaker and the resistance changes linearly with voltage approximately. (C) 2013 American Institute of Physics. [http://dx.doi.org/10.1063/1.4796050]
\end{abstract}

Perovskite manganese oxides have attracted continuing attention for decades due to their striking magnetic and electric properties. ${ }^{1-3}$ Such materials can exhibit huge responses to applied magnetic fields. ${ }^{4,5}$ The great sensitivity largely originates from the cross coupling between different physical degrees of freedoms (charge, spin, orbital, and lattice). One manifestation of the complex coupling is that manganites can be affected in a number of ways: chemical doping, pressure, light illumination, magnetic field, electric field, lattice strain, and etc. $^{6-9}$ Since the lattice strain is frequently present in the growth of thin films, it is very important to understand the strain effects. Conventionally, to study the strain effects, one can compare films with various thicknesses on the same substrate or with identical thickness on different substrates. ${ }^{10-12}$ Varying thickness could tune the lattice parameter in a small range. Changing substrates could make the strain state be discrete values in a wider range. One concern about these two methods is that the variation of a second parameter, such as oxygen nonstoichiometry may have sizeable effects, ${ }^{13,14}$ which are hard to quantify. Employing the converse piezoelectric effect of piezoelectric substrates is a recently developed route to evaluate the strain effects. ${ }^{15-19}$ Its achievable strain range is not large, but the strain can be continuously and reversibly tuned. Considering the advantages and disadvantages of these methods, a combination of them may give valuable information. For manganites, $0.7 \mathrm{PbMg}_{1 / 3} \mathrm{Nb}_{2 / 3}$ $\mathrm{O}_{3}-0.3 \mathrm{PbTiO}_{3}$ (PMN-PT) substrate is frequently chosen for it possesses a pervoskite structure and high piezoelectric coefficients, which depend weakly on temperature. ${ }^{20,21}$ For PMNPT, the lattice constant a $\sim 4.020 \AA$. It is much larger than that for all manganites (pseudocubic, $a_{\text {manganite }}<3.920 \AA$ ). The large lattice mismatch usually leads to a fast strain relaxation. ${ }^{16,22}$ Among all colossal magnetoresistance (CMR) manganites, LaBaMnO have the

\footnotetext{
${ }^{\text {a) }}$ Author to whom correspondence should be addressed. Electronic mail: jugao@hku.hk
}

largest lattice constants. Thus the lattice mismatch between LaBaMnO and PMN-PT should be smaller than that between PMN-PT and other manganites. It is of interest to study the behaviors of LaBaMnO films on PMN-PT. In this letter, we present a detailed study on strain effects on transport properties of $\mathrm{La}_{0.7} \mathrm{Ba}_{0.3} \mathrm{MnO}_{3}$ (LBMO) thin films. The effects of residual strain were studied by comparing films with various thicknesses on two different substrates, PMN-PT and $\mathrm{SrTiO}_{3}$ (STO). The tunable strain was controlled through the converse piezoelectric effects of PMN-PT.

LBMO films with different thicknesses $(t=15,30,60$, and $100 \mathrm{~nm}$ ) were grown on (001) oriented single crystalline substrates of PMN-PT and STO by using pulsed laser deposition. During deposition, the substrates were kept at $680^{\circ} \mathrm{C}$ in oxygen with a pressure of $70 \mathrm{~Pa}$. Samples were annealed in situ at grown temperature in oxygen $\left(10^{5} \mathrm{~Pa}\right)$. The silver $(\mathrm{Ag})$ electrodes on the surface of LBMO films and the back of PMN-PT substrates were deposited by thermal evaporation. A Keithley 6487 voltage source was used to apply the dc voltage across PMN-PT. The leakage currents in PMN-PT were negligible (less than $10 \mathrm{nA}$ ).

For all films on PMN-PT, only reflection peaks from (001) crystal plane of LBMO and PMN-PT can be found from the XRD patterns $(\theta-2 \theta$ scan). Figures $1(a)$ and $1(b)$ show such patterns in the vicinity of (002) reflection peaks of LBMO. For the $15 \mathrm{~nm}$-thick film, the out-of-plane lattice constant, which is calculated from the reflection peak, is $\mathrm{c} \sim 3.862 \AA$, indicating compressive out-of-plane strains $\left[\varepsilon_{\mathrm{zz}}=\left(\mathrm{c}_{\text {film }}-\mathrm{c}_{\text {bulk }}\right) / \mathrm{c}_{\text {bulk }}\right]$ of $\sim-1.38 \%$. The in-plane strain $\varepsilon_{\mathrm{xx}}$ may be estimated by $\varepsilon_{\mathrm{XX}}=-\varepsilon_{\mathrm{zz}}(1-\nu) /(2 \nu)$, where $\nu$ is the Poisson ratio. For manganites, the typical values of $\nu$ range between 0.3 and 0.5 . Thus, $\varepsilon_{\mathrm{XX}}$ should be no larger than $1.61 \%$. The lattice mismatch $\mathrm{f}_{\text {mis }}$ between bulk LBMO and PMN-PT can be easily calculated $\mathrm{f}_{\text {mis }}=\left(\mathrm{a}_{\text {PMN-PT }}-\mathrm{a}_{\text {LBMObulk }}\right) / \mathrm{a}_{\text {PMN-PT }} \sim 2.58 \%$. In other words, the strain in the LBMO film is partially relaxed. The process of strain relaxation in LBMO (on PMN-PT) with film thickness is obviously different from that in $\operatorname{Pr}_{0.7} \mathrm{Sr}_{0.3} \mathrm{MnO}_{3}$ 

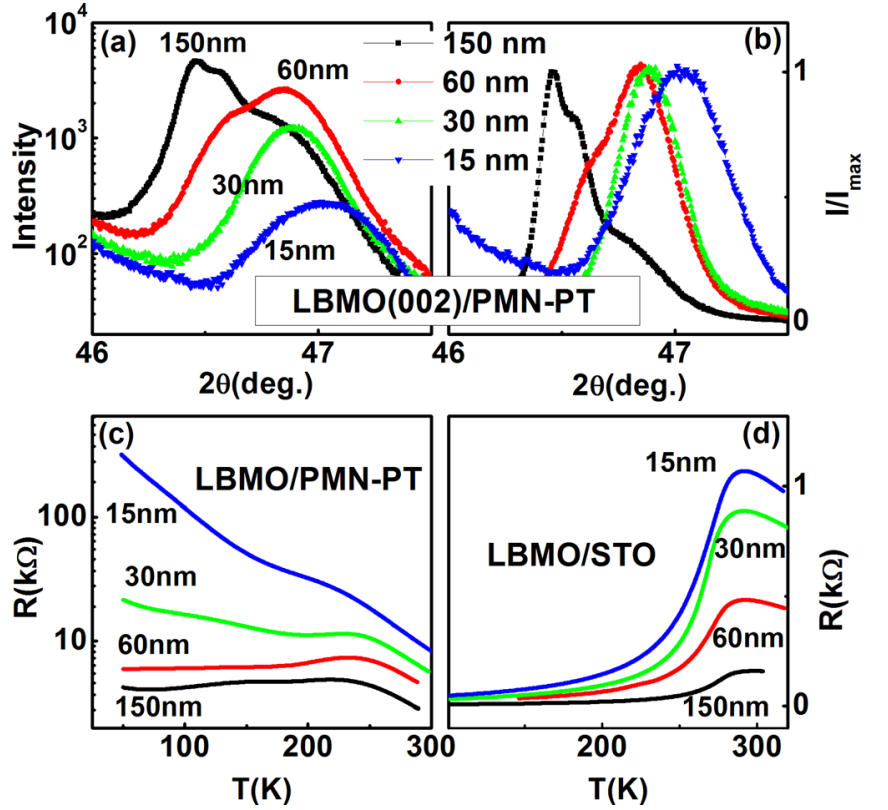

FIG. 1. (a) and (b): X-ray diffraction patterns [in the vicinity of (002) reflection peaks of LBMO] for LBMO films with different thicknesses ( $t=15,30$, 60 , and $150 \mathrm{~nm}$ ) on PMN-PT. The curves in (a) are as-measured and in (b) are normalized by the maximum peak intensities; (c) and (d) R(T) curves of LBMO films on PMN-PT (c) and STO (d).

(PSMO, on PMN-PT). ${ }^{22}$ There are clear evolutions of peak (intensity, position, and shape) with thickness. With the increase of film thickness, the diffraction peak becomes more asymmetric. This behavior is possibly due to the inhomogenous strain as a result of strain relaxation. ${ }^{23}$ As the thickness increases from $15 \mathrm{~nm}$ to $150 \mathrm{~nm}$, the out-of-plane lattice parameter increases from $\sim 3.862 \AA$ to $\sim 3.901 \AA$. At the same time, $\mathrm{R}(\mathrm{T})$ curves also changes dramatically [see Fig. 1(c)]. For LBMO on PMN-PT with a thickness of $15 \mathrm{~nm}$, the resistivity is greatly enhanced. As the temperature is lowered, the resistance increased monotonously. No metal-to-insulator transition can be found. The resistance in thicker films is smaller in the whole temperature range. For LBMO on PMN-PT with thicknesses of $60 \mathrm{~nm}$ and $150 \mathrm{~nm}$, clear metal-to-insulator transition can be seen. For film with an intermediate thickness $(\mathrm{t}=30 \mathrm{~nm})$, with decreasing temperature, there is an insulatorto-metal transition slightly below room temperature and a low-temperature upturn of resistance in the $\mathrm{R}(\mathrm{T})$ curve.

For all LBMO films on STO, there are strong overlaps of reflection peaks for LBMO and STO (not shown). This is because the lattice parameter of bulk LBMO ( $\left.\mathrm{a}_{\text {LBMOps }} \sim 3.916 \AA\right)$ is very close to that of STO $\left(\mathrm{a}_{\mathrm{STO}} \sim 3.905 \AA\right)$. As a result of such overlaps, it is difficult to obtain the evolutions of peak intensity, as well as position and shape, when the thickness varies. As shown in Fig. 1(d), all LBMO films on STO have a clear metal-to-insulator transition near room temperatures, which does not change much as the thickness varies. The transition temperatures are close to that reported by Zhang et al. ${ }^{24}$ Compared to that on PMN$\mathrm{PT}$, the resistivity of LBMO on STO is much smaller. Since LBMO with the same thickness on PMN-PT and STO were deposited with identical parameters, the differences in transport properties should be mainly due to the effects of residual strain.

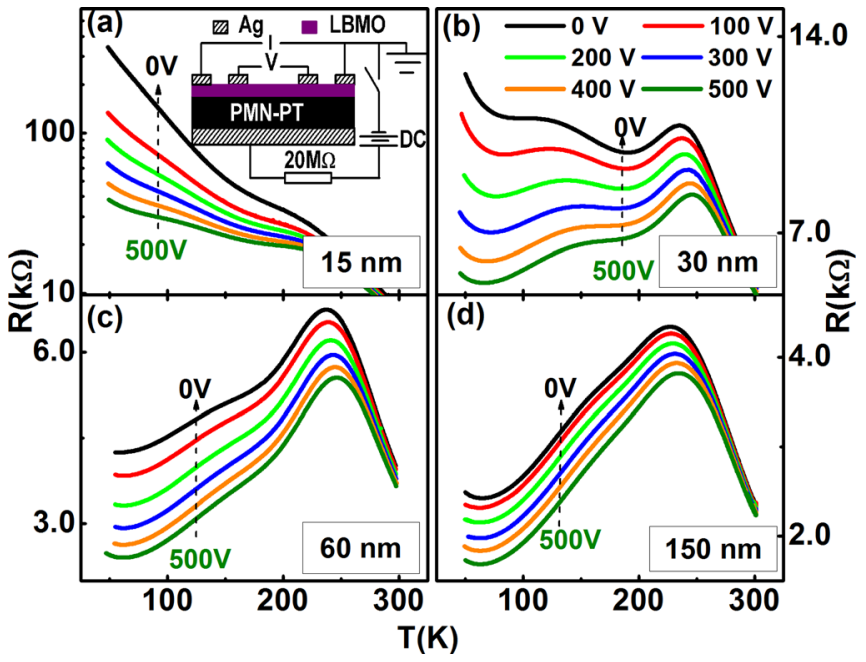

FIG. 2. R(T) curves for LBMO films with different thicknesses under different bias voltages (from $500 \mathrm{~V}$ to $0 \mathrm{~V}$, the voltage interval is $100 \mathrm{~V}$ ). The inset of (a): A schematic diagram of measurement circuit.

The schematic diagram for measuring transport properties is depicted in the inset of Fig. 2(a). The polarity in the schematic diagram is defined as positive. PMN-PT was poled by a $+500 \mathrm{~V}$ voltage $(\mathrm{E}=10 \mathrm{kV} / \mathrm{cm})$ across PMN-PT. It was in the $\mathrm{P}_{\mathrm{R}}{ }^{+}$state after the poling electric field was removed. To study the effects of tunable strain, the temperature dependences of resistance for $\mathrm{LBMO}$ were recorded with different positive bias voltages. As shown in Fig. 2, for all LBMO films, clear changes can be found in the R-T curves as the voltage varied. It is interesting to note that an electric field of $+10 \mathrm{kV} / \mathrm{cm}(\mathrm{V}=500 \mathrm{~V})$ almost completely suppressed the upturn of resistance at low temperatures [see Fig. 2(b)].

The temperature dependences of resistance modulation under a fixed voltage, $\delta(\mathrm{V})=|\Delta \mathrm{R}| / \mathrm{R}=|\Delta \mathrm{R}(\mathrm{V})| / \mathrm{R}(0)=100 \%$ $\times|[\mathrm{R}(\mathrm{V})-\mathrm{R}(0)]| / \mathrm{R}(0)$, are presented in Fig. 3. There are clear differences for LBMO and PSMO. While for all PSMO films, $|\Delta \mathrm{R}| / \mathrm{R}$ peaks are at a temperature slightly lower than $\mathrm{T}_{\mathrm{P}},{ }^{22}$ for all LBMO films $|\Delta \mathrm{R}| / \mathrm{R}$ increases as temperature decreases. Although there are clear metal-to-insulator transitions in LBMO films with thicknesses of 60 and $150 \mathrm{~nm}$, only

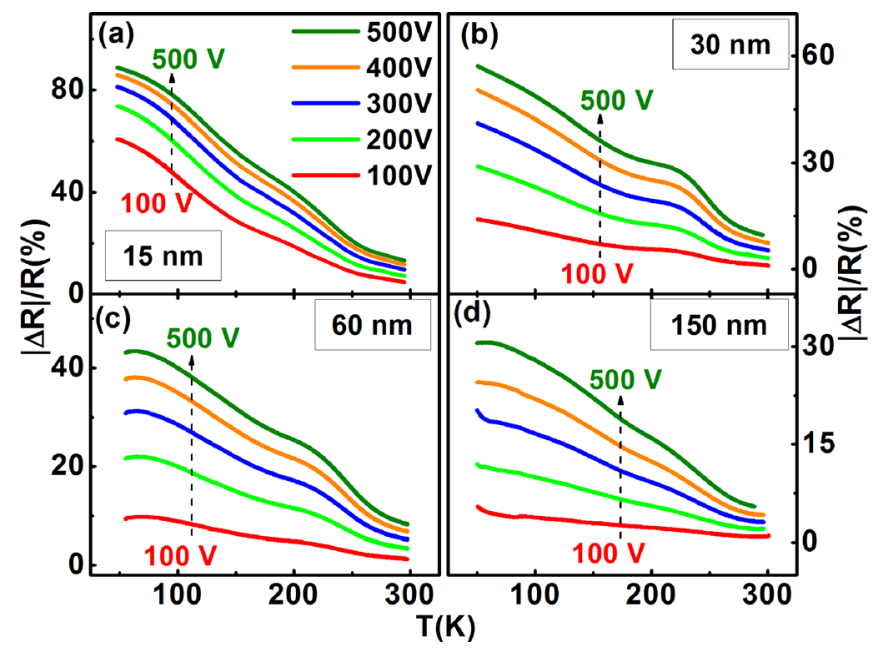

FIG. 3. Temperature dependences of resistance modulation, $|\Delta R(V)| /$ $\mathrm{R}(0)=|[\mathrm{R}(\mathrm{V})-\mathrm{R}(0)]| / \mathrm{R}(0)$, for LBMO/PMN-PT with different thicknesses. 


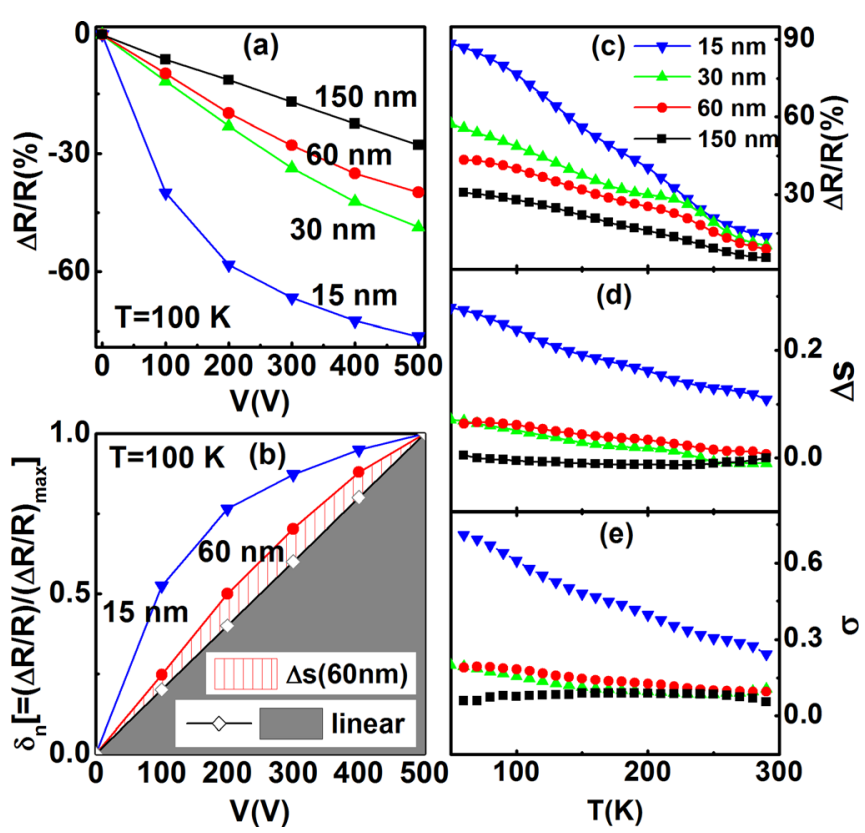

FIG. 4. (a) The dependences of relative resistance change $|\Delta \mathrm{R}(\mathrm{V})| / \mathrm{R}(0)$ on bias voltage at $100 \mathrm{~K}$; (b): Normalized resistance modulation, $\delta_{\mathrm{n}}(\mathrm{V})=\delta(\mathrm{V}) /$ $\delta(500 \mathrm{~V})$, as a function of applied voltages when the voltage was decreased from $500 \mathrm{~V}$ to $0 \mathrm{~V}$ with a step of $100 \mathrm{~V}(\mathrm{~T}=100 \mathrm{~K}, \mathrm{t}=15$, and $60 \mathrm{~nm})$. For comparison, a linear dependence is shown; (c)-(e): Temperature dependence of resistance modulation $|\Delta \mathrm{R}| / \mathrm{R}$ (c), area difference $\Delta \mathrm{s}$ (d), and standard deviation $\sigma$ (e) for LBMO with different thicknesses.

anomaly but no clear peak is found in the temperature dependences of $|\Delta R| / R$. There are also several similarities for LBMO and PSMO. ${ }^{22}$ The relative resistance changes $|\Delta R| / R$ in the thinnest film is the largest. For LBMO with a thickness of $15 \mathrm{~nm}$, the maximum value of $|\Delta \mathrm{R}| / \mathrm{R}$ is $\sim 90 \%$ at low temperatures. With increasing thicknesses, $|\Delta \mathrm{R}| / \mathrm{R}$ becomes smaller. For the film with a large thickness, e.g., $t=150 \mathrm{~nm},|\Delta \mathrm{R}| / \mathrm{R}$ is still considerable. Another prominent similarity is that, for films with small thicknesses, the change of resistance as the voltage decreases from $100 \mathrm{~V}$ to $0 \mathrm{~V}$ is different from that when the voltage drops from $500 \mathrm{~V}$ to $0 \mathrm{~V}$. This means a nonlinear relation between $|\Delta \mathrm{R}| / \mathrm{R}$ and $\mathrm{V}$.

The nonlinearity can be clearly seen from the voltage dependence of normalized resistance change, $\delta_{\mathrm{n}}=\delta(\mathrm{V}) /$ $\delta(500 \mathrm{~V})$, at selected temperatures. ${ }^{22}$ Figures $4(\mathrm{a})$ and $4(\mathrm{~b})$ plot $|\Delta \mathrm{R}| / \mathrm{R}$ and $\delta_{\mathrm{n}}$ as a function of applied voltage at $100 \mathrm{~K}$. To get some insights, we use two quantities defined in Ref. 22, the additional area $\Delta s$ and the standard deviation $\sigma$, to characterize the nonlinearity. For the convenience of calculation, the voltage was normalized by $500 \mathrm{~V}$. The additional area $\Delta s$ can be determined from the formula, $\Delta s=0.2 \sum_{i=1}^{5} \delta_{n}(100 i)-0.6$. The standard deviation $\sigma$ examines the deviation from a linear $\delta_{\mathrm{n}}-\mathrm{V}$ curve, $\sigma=\sqrt{\sum_{i=1}^{5}\left[\delta_{n}(100 i)-0.2 i\right]^{2}}$. For a linear resistancevoltage relation, both $\Delta \mathrm{s}$ and $\sigma$ should be 0 . The strong nonlinearity in LBMO with a thickness of $15 \mathrm{~nm}$ is evidenced from the large values of $\Delta s$ and $\sigma$ at all temperatures. For
LBMO films with larger thicknesses, the nonlinearity is less significant. The temperature dependences of $|\Delta R / R|, \Delta s$, and $\sigma$ are also very similar, suggesting close relations between these quantities. The similarities in temperature evolutions of $|\Delta \mathrm{R} / \mathrm{R}|, \Delta \mathrm{s}$, and $\sigma$ were observed in PSMO, ${ }^{22}$ where it is suggested that phase competition significantly enhance both the magnitude and nonlinearity of elastoresistance. For LBMO studied in this paper, the increase of $|\Delta R / R|$ at low temperatures cannot be attributed to the enhancement of piezoelectric strain, which decreases slightly while the temperature is lowered. ${ }^{21}$ Possibly, the simultaneous rises in $|\Delta \mathrm{R} / \mathrm{R}|, \Delta \mathrm{s}$, and $\sigma$ during cooling reflect more prominent phase competition at lower temperatures. It appears that the residual strain favors an insulating conduction and considerable insulating parts preserved even at low temperatures. This may be the reason that there is no peaks in temperature dependence of $|\Delta \mathrm{R} / \mathrm{R}|, \Delta \mathrm{s}$, and $\sigma$.

To conclude, the effects of residual and tunable strain on the transport properties in thin films of LBMO are studied. For thin films of LBMO on PMN-PT, both the XRD reflection peaks and temperature dependence of resistance evolve dramatically when the thickness varies. On contrary, the transport properties in LBMO films on STO have no substantial dependence on thickness. Tunable strain effects in LBMO/PMN-RT were studied by employing the converse piezoelectric effects of PMN-PT. Clear resistance modulations were observed. For thinnest LBMO film, the strong nonlinearity is evidenced from the large values of $\Delta \mathrm{s}$ and $\sigma$. The nonlinearity becomes weaker with increasing thicknesses. There are similarities in the temperature dependences of $|\Delta \mathrm{R}| / \mathrm{R}, \Delta \mathrm{s}$, and $\sigma$ for LBMO on PMN-PT.

This work has been supported by a grant of the Research Grant Council of Hong Kong (Project No. HKU 702112 P) and CRCG of HKU.

\footnotetext{
${ }^{1}$ E. Dagotto et al., Phys. Rep. 344, 1 (2001).

${ }^{2}$ M. B. Salamon and M. Jaime, Rev. Mod. Phys. 73, 583 (2001).

${ }^{3}$ Y. Tokura, Rep. Prog. Phys. 69, 797 (2006).

${ }^{4} \mathrm{R}$. von Helmolt et al., Phys. Rev. Lett. 71, 2331 (1993).

${ }^{5}$ S. Jin et al., Science 264, 413 (1994).

${ }^{6}$ J. R. Sun et al., Phys. Rev. B 67, 144414 (2003).

${ }^{7}$ S. Arumugam et al., J. Appl. Phys. 107, 113904 (2010).

${ }^{8}$ J. F. Wang and J. Gao, J. Appl. Phys. 109, 07d701 (2011).

${ }^{9}$ J. F. Wang, Z. P. Wu, and J. Gao, J. Appl. Phys. 111, 07 E131 (2012).

${ }^{10}$ F. Yang et al., Appl. Phys. Lett. 97, 092503 (2010).

${ }^{11}$ C. Adamo et al., Appl. Phys. Lett. 95, 112504 (2009).

${ }^{12}$ W. Prellier et al., Appl. Phys. Lett. 75, 397 (1999).

${ }^{13}$ P. Murugavel et al., Appl. Phys. Lett. 82, 1908 (2003).

${ }^{14}$ J. Dvorak et al., J. Appl. Phys. 97, 10C102 (2005).

${ }^{15}$ D. Dale et al., Appl. Phys. Lett. 82, 3725 (2003).

${ }^{16}$ C. Thiele et al., Appl. Phys. Lett. 87, 262502 (2005).

${ }^{17}$ R. K. Zheng et al., Appl. Phys. Lett. 92, 082908 (2008).

${ }^{18}$ M. D. Biegalski et al., Appl. Phys. Lett. 96, 151905 (2010).

${ }^{19}$ E. J. Guo, J. Gao, and H. B. Lu, Appl. Phys. Lett. 98, 081903 (2011).

${ }^{20}$ S.-E. Park and T. R. Shrout, J. Appl. Phys. 82, 1804 (1997).

${ }^{21}$ A. Herklotz et al., J. Appl. Phys. 108, 094101 (2010).

${ }^{22}$ J. F. Wang and J. Gao, Appl. Phys. Lett. 100, 131903 (2012).

${ }^{23}$ G. H. Aydogdu et al., Mater. Sci. Eng. B 144, 123 (2007).

${ }^{24}$ J. Zhang et al., Phys. Rev. B 64, 184404 (2001).
} 